\title{
Numerical Simulation and Experiment of the Effects of Blade Angle Deviation on the Hydraulic Characteristics and Pressure Pulsation of an Axial-Flow Pump
}

\author{
Lijian Shi $\oplus^{1},{ }^{1}$ Jun Zhu, ${ }^{1}$ Yao Yuan, ${ }^{2}$ Fangping Tang, ${ }^{1}$ Penglan Huang, ${ }^{3}$ Wenpeng Zhang, \\ Haiyu Liu, ${ }^{1}$ and Xiaowen Zhang ${ }^{1}$ \\ ${ }^{1}$ College of Hydraulic Science and Engineering, Yangzhou University, Yangzhou 225000, Jiangsu, China \\ ${ }^{2}$ Jiangsu Hydraulic Research Institute, Nanjing 210000, Jiangsu, China \\ ${ }^{3}$ Yangzhou Municipal Pipe Network Co., Ltd., Yangzhou 225000, Jiangsu, China
}

Correspondence should be addressed to Lijian Shi; shilijian@yzu.edu.cn

Received 2 November 2020; Revised 3 December 2020; Accepted 19 December 2020; Published 4 January 2021

Academic Editor: Ling Zhou; lingzhou@ujs.edu.cn

Copyright (c) 2021 Lijian Shi et al. This is an open access article distributed under the Creative Commons Attribution License, which permits unrestricted use, distribution, and reproduction in any medium, provided the original work is properly cited.

Inadequate blade angle adjustment or manufacturing errors will cause inconsistencies in the blade angle of an axial-flow pump. In this study, the hydrodynamic characteristics of an axial-flow pump with inconsistent blade angle are investigated by analyzing hydraulic performance and pressure pulsation. The analysis is conducted by performing a numerical simulation combined with a model test. Results show that, relative to the case without blade angle deviation, the case with blade angle deviation exhibits changes in the periodicity of the flow field in the impeller. Such changes result in uneven pressure changes in the impeller passage. The pressure pulsation induced by the blade angle deviation is mainly low-frequency pulsation; that is, it is twice the rotation frequency. The amplitude of the main frequency pulsation is 1.5-3 times that of the blade without angle deviation. This low frequency that dominates the whole pump device easily causes the vibration and weakens the safety and stability of the pump. The blade angle deviation exerts great influence on the unsteady characteristics. Hence, blade angle deviation seriously affects the safe and stable operation of axial-flow pumps and pump stations.

\section{Introduction}

Axial-flow pumps are characterized by a low head and large flow, and they mainly rely on the rotation of impellers to convert the work into the kinetic energy and pressure energy of water. These pumps are widely used in the East Route Project of the South-to-North Water Diversion in China and they play an important role in agricultural irrigation, urban water supply, and drainage [1-6]. At present, large-scale pump station projects generally adopt fully adjustable impellers. Blade angles are adjusted in time according to the operation requirements to achieve energy-saving and safe operations. In recent years, with the development of computer technology and CFD technology, the numerical simulation has become an important means to analyze the internal flow and hydraulic characteristics of pump devices
[7-10]. Many experts and scholars at home and abroad have studied the influence of blade angle on pump devices, and they have mainly focused on the effects of blade angle changes on the saddle zone characteristics, cavitation characteristics, and unsteady characteristics of pump device [11-16]. References [17-19] used the numerical simulation to study the effects of impeller outlet angle on the hydraulic performance, vibration characteristics, and noise of centrifugal pumps. Wang et al. [20] explored that the influence of the variation law of blade release angle on turbine hydraulic performance. Cui et al. [21] found that the suitable cutting of the blade tailing angle can improve the hydraulic performance, unstable flow, and vibration of the centrifugal pump. Liu et al. [22] found that with an increase in the blade rotation angle, the best efficiency point shifts toward a large flow condition under the pump mode and turbine mode; the 
authors also analyzed the evolution of the tip leakage vortex when the blade rotation angles are $4^{\circ}$ and $-4^{\circ}$. Peng et al. [23] showed that increasing the blade outlet angle pair reduces the cavitation performance of a multistage submersible pump with a low specific speed and increase the flow separation of the blade working surface. Feng et al. [24] set up a mathematical model based on the basis of the minimum daily operating cost of a pump station and summarized the influence of adjusting the blade angle frequency on the optimal operation of the pump station; they proposed to adjust the blade angles four to six times a day. Wei et al. [25] analyzed the influence of the inlet angle of a diffuser blade on the performance of a submersible pump and found that the maximum power point moves toward a large flow with an increase in blade inlet angle and that the range of the highefficiency zone becomes wide. Kim et al. [26] performed a multidisciplinary optimization of a submersible axial-flow pump by using a hybrid multiobjective evolutionary algorithm and adopting the impeller's sweep angle and pitch angle as the design variables; the results showed the improved hydraulic performance of the pump. For a fully regulated impeller, the angle of each blade may vary if the adjustment is not smooth during the blade adjustment process. As the flow in the impeller passage is complex turbulent motion, it affects the safe operation and overall hydraulic performance of a pump. Bing and Cao [27, 28] found that a partial rotation deviation of a mixed-flow pump affects the performance of the pump device and reduces the symmetry and uniformity of the pressure distribution in the mixed-flow pump. The above mainly introduces the research results of the blade angle, and there are many research [29-31] results on the hydraulic characteristics, internal flow mechanism, and pulsation characteristics of the pump.

Blade angles are not always adjusted properly, and manufacturing errors are inevitable in axial-flow pumps. These conditions lead to blade angle deviations in axial-flow pumps. In the current work, the numerical simulation and the model test are combined to study the characteristics of the internal and external flow of a pump device and its pressure pulsation under blade angle deviation. The results are expected to improve the understanding of blade angle deviation and serve as a reference for the safe and stable operation of pumps.

\section{Numerical Calculation}

2.1. Governing Equations. A continuous equation is applied in this work on the basis of the finite volume method. The pump's inner flow field, that is three-dimensional incompressible turbulent flow, is described by the Navier-Stokes equation. The turbulent model adopts $k-\varepsilon$ turbulence model, which modifies turbulent viscosity considering the average flow of rotation and rotation flow, and can better handle the high strain rate and degree of streamline curvature flow.

In the standard $k-\varepsilon$ turbulence model, $k$ and $\varepsilon$ are two basic unknowns, and the corresponding transport equation is

$$
\begin{aligned}
& \frac{\partial(\rho k)}{\partial t}+\frac{\partial\left(\rho k u_{i}\right)}{\partial x_{i}}=\frac{\partial}{\partial x_{j}}\left[\left(\mu+\frac{\mu_{t}}{\sigma_{k}}\right) \frac{\partial k}{\partial x_{j}}\right]+G_{k}+G_{b}-\rho \varepsilon-Y_{M}+S_{k}, \\
& \frac{\partial(\rho \varepsilon)}{\partial t}+\frac{\partial\left(\rho \varepsilon u_{i}\right)}{\partial x_{i}}=\frac{\partial}{\partial x_{j}}\left[\left(\mu+\frac{\mu_{t}}{\sigma_{k}}\right) \frac{\partial \varepsilon}{\partial x_{j}}\right]+C_{1 \varepsilon} \frac{\varepsilon}{\mathrm{k}}\left(G_{k}+C_{3 \varepsilon} G_{b}\right)-C_{2 \varepsilon} \rho \frac{\varepsilon^{2}}{k}+S_{\varepsilon},
\end{aligned}
$$

where $G_{k}$ is the generation term of turbulent kinetic energy $k$ caused by average velocity gradient, $G_{b}$ is the generation term of turbulent kinetic energy $k$ caused by buoyancy, $Y_{M}$ is the contribution of pulsation expansion in compressible turbulent pulsation, $C_{1 \varepsilon}, C_{2 \varepsilon}$, and $C_{3 \varepsilon}$ are empirical constants, $\sigma_{k}$ and $\sigma_{\varepsilon}$ are the Prandtl numbers corresponding to turbulent kinetic energy $k$ and dissipation rate $\varepsilon$, respectively, and $S_{k}$ and $S_{\varepsilon}$ are the user-defined source terms.

2.2. Calculation Model. Figure 1 shows the three-dimensional turbulence model of the pump device in this work. The numerical simulation domain mainly includes the inlet pipe, inlet cone pipe, impeller, guide vane, and outlet pipe. The calculated parameters of the pump are as follows: the number of impeller blades is 4 , the number of guide vane blades is 7 , the rotation speed $(n)$ is $1450 \mathrm{r} / \mathrm{min}$, the diameter of the impeller $(D)$ is $300 \mathrm{~mm}$, the hub ratio $\left(d_{h}\right)$ is 0.4 , and the design flow $\left(Q_{d}\right)$ is $360 \mathrm{~L} / \mathrm{s}$. Tip clearance is not considered in the calculation.

2.3. Grid Independence Analysis. Unstructured grid division is applied to the axial-flow pump's impeller, whereas structured grid division is applied to the inlet pipe, inlet cone pipe, guide vane, and outlet pipe. Local grid encryption is carried out on the twisted parts, such as the impeller and guide vane, to ensure the accuracy of the numerical simulation. As the degree of grid density exerts great influence on the accuracy of the numerical simulation, this work analyzes the grid independence of the impeller and takes efficiency as the index of the grid independence evaluation (Figure 2). As shown in Figure 2, when the grid in the impeller increases to 2.4 million, the pump efficiency fluctuates steadily with the increase of the grid number, and the relative efficiency changes within $1 \%$, thus meeting the requirements of grid independence. Therefore, this study selects 2.4 million 


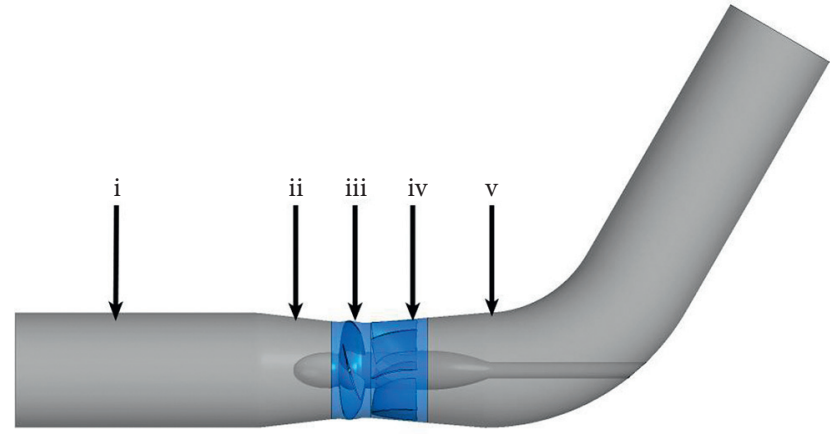

Figure 1: Axial-flow pump device model. (i) Inlet pipe. (ii) Inlet cone pipe. (iii) Impeller. (iv) Guide vane. (v) Outlet pipe.

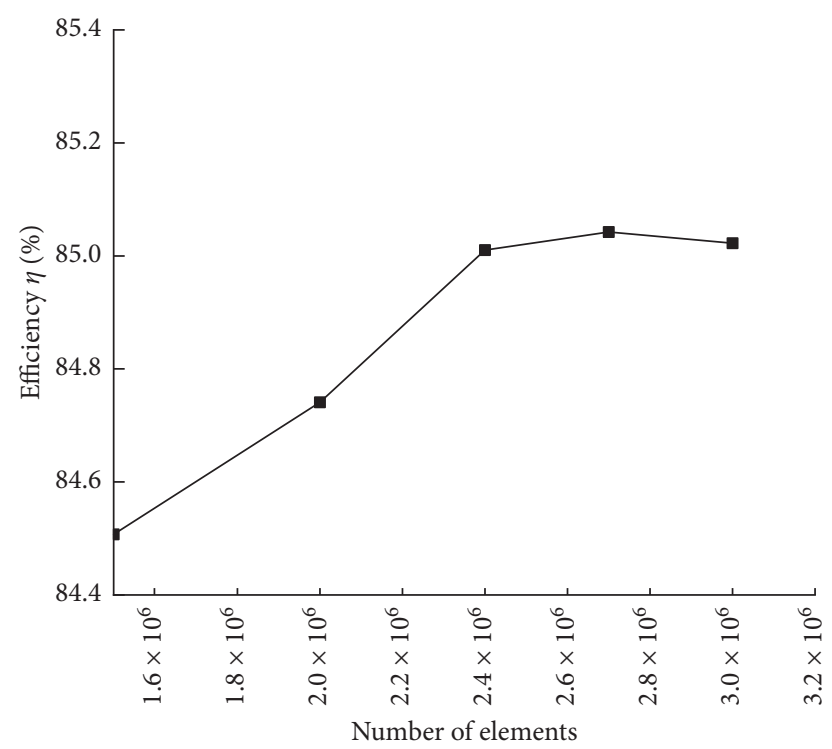

Figure 2: Grid independence analysis.

impeller grids on the basis of the results of the grid independence analysis (Figure 3).

2.4. The Boundary Conditions. In the steady calculation, the inlet condition is set to the total pressure with a value of $1 \mathrm{~atm}$, and the outlet boundary is set to mass flow. The standard wall function is used in the near-wall area, while the wall is set to have no-slip condition. The interface between the impeller and the guide vane is set to a "stage model" with the average circumferential speed. Transient simulation calculation is performed on the basis of the steady calculation results. The interface between the impeller and the guide vane in the unsteady calculation is set to a "transient rotor interface model." Each time step of unsteady calculation, that is, the time required for the impeller to rotate by $3^{\circ}$, is $3.45 \times 10^{-4} \mathrm{~s}$. The total time refers to the time required for the impeller rotating 6 revolutions.

To ensure the convergence of the numerical simulation results, this study takes the change of efficiency monitoring value as the judgment basis (Figure 4). Figure 4 shows the curve of the efficiency monitoring point with the number of iterative steps. When the number of iterative steps reaches
1,000 , the efficiency monitoring value tends to be stable. By taking computer performance and other factors into consideration, this study sets the number of iterative steps to 1,200 .

2.5. Scheme Setting. In this paper, blade angle deviation refers to the inconsistency in the changes in blade angle during blade angle adjustment. In the absence of deviation, the angles of the four blades are consistent, as shown in Figure 5(a). The direction of blade adjustment is positive in the counterclockwise direction around the center of the blade chord (Figure 5(b)). The blade angle deviation in this work is set as symmetrical deviation; that is, the angles of two symmetrical blades are the same. This study mainly focuses on the steady and unsteady characteristics of a pump device with symmetrical blade angle deviation and without blade angle deviation. The scheme design is shown in Table 1.

\section{Model Test}

In this study, an axial-flow pump device is taken as the test object. The physical model includes an inlet pipe, inlet cone pipe, impeller, guide vane, $60^{\circ}$ elbow pipe, and outlet pipe. The number of blades is 4 , the number of guide vanes is 7 , the blade angle is $0^{\circ}$, the impeller diameter $(D)$ is $300 \mathrm{~mm}$, the hub ratio $\left(d_{h}\right)$ is 0.4 , and the tip clearance is controlled within $0.2 \mathrm{~mm}$. The model diagram of the impeller, guide vane, and pump device is shown in Figure 6.

The physical model test on the pump device was conducted on a high-precision hydraulic machinery test bench of the Key Laboratory of Hydraulic and Power Engineering. The device is a vertical closed circulation system. No less than 18 performance test points are collected. The comprehensive error of the efficiency test system is $\pm 0.39 \%$. The reliability of the numerical simulation results of the pump device is verified accordingly.

Figure 7 compares the performances of the pump device predicted by the numerical simulation and model test with four blade angles of $0^{\circ}$. The numerical simulation results are basically consistent with the overall trend of the model test. Under the design condition, the head of the numerical simulation is $5.78 \mathrm{~m}$, and the efficiency is $85.01 \%$. The model test head is $5.29 \mathrm{~m}$, of which the head error is $0.49 \mathrm{~m}$; and the efficiency is $84.5 \%$, of which the efficiency error is $0.51 \%$. The difference between the numerical simulation and the model test is small, thus indicating that the numerical simulation results have high accuracy and that the calculation results are credible. At the same time, because of the existence of tip clearance in the test, the head and efficiency errors under the off-design conditions are large. In sum, the numerical simulation results under the design conditions are reliable.

\section{Results and Discussion}

4.1. Energy Performance Prediction. The pump head is calculated by the Bernoulli equation, and the pump performance is predicted by the torque acting on the blade and hub.

The head equation is shown as follows: 


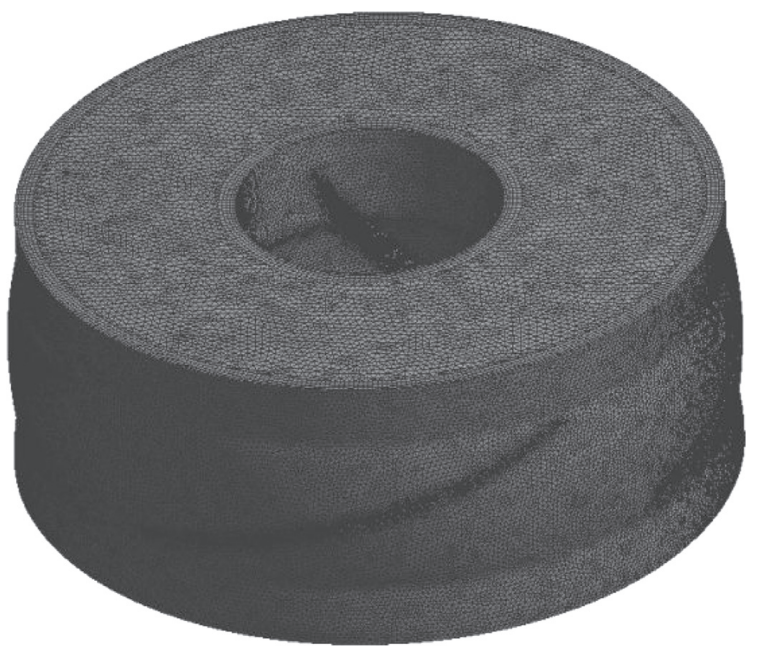

FIgURE 3: The grid of the impeller.

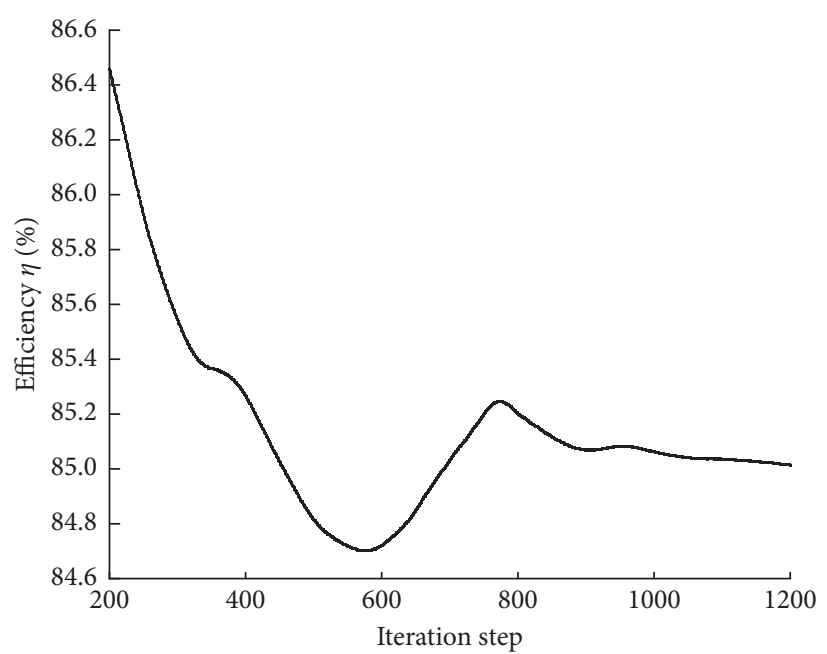

FIGURE 4: Efficiency curve with iteration step.

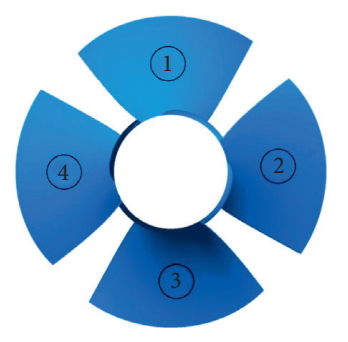

(a)

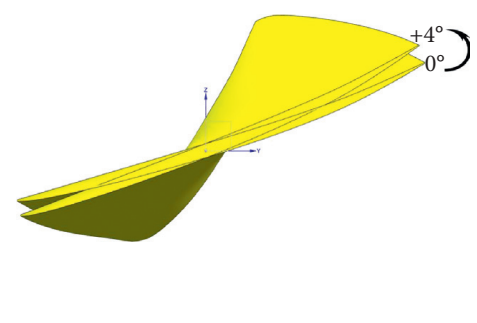

(b)

Figure 5: The blade angle distribution. (a) The label of the blade. (b) Blade angle figure.

$$
H=\frac{\left(P_{\text {out }}-P_{\text {in }}\right)}{\rho g},
$$

where $P_{\text {out }}$ is the total pressure at outlet section of pump device, $\mathrm{Pa} ; P_{\text {in }}$ is the total pressure at inlet section of pump device, $\mathrm{Pa}$; and $g$ is the local acceleration of gravity, $\mathrm{m} / \mathrm{s}^{2}$.

The efficiency equation is shown as follows:
TABLE 1: Design scheme table.

\begin{tabular}{lccccc}
\hline \multirow{2}{*}{ Scheme number } & \multicolumn{5}{c}{ Blade angle $\left(^{\circ}\right)$} \\
& 1 & 2 & 3 & 4 & \\
\hline I & 0 & 0 & 0 & 0 & No deviation \\
II & 0 & +4 & 0 & +4 & Symmetrical deviation \\
III & +2 & +2 & +2 & +2 & No deviation \\
IV & +4 & +4 & +4 & +4 & No deviation \\
\hline
\end{tabular}




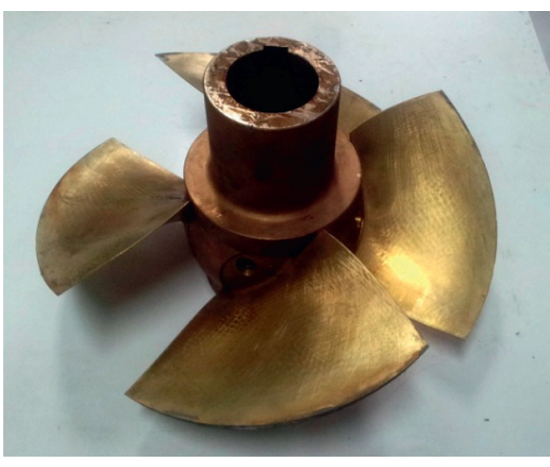

(a)

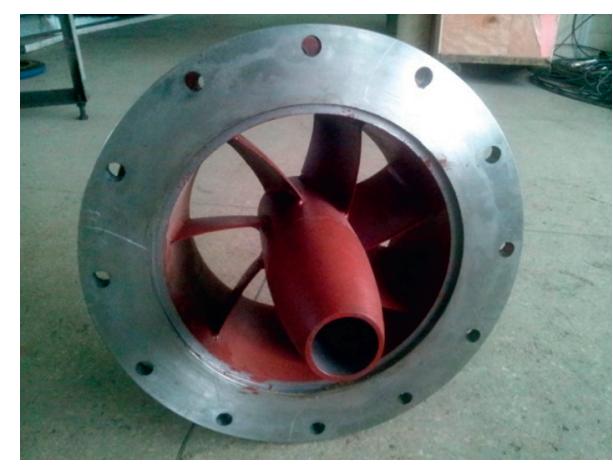

(b)

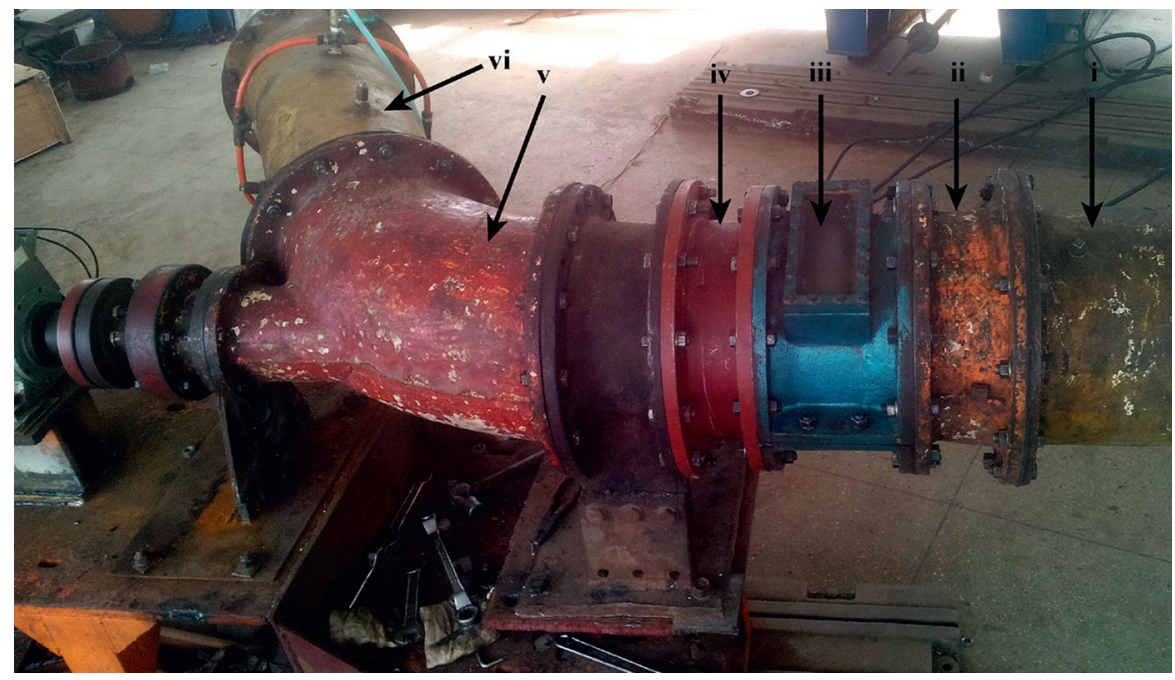

(c)

Figure 6: Model device. (a) Impeller. (b) Guide vane. (c) Pump installation with (i) inlet pipe, (ii) inlet cone pipe, (iii) impeller, (iv) guide vane, (v) $60^{\circ}$ elbow pipe, and (vi) outlet pipe.

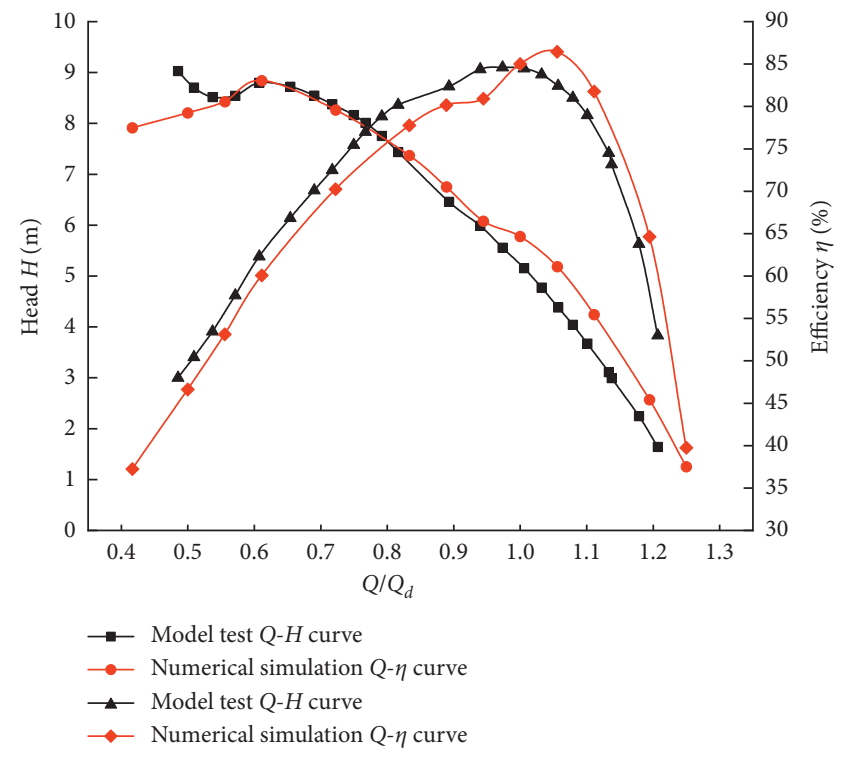

FIGURE 7: Performance comparison of numerical simulation and model test.

$$
\eta=\frac{\rho g Q H}{T_{p} \omega},
$$

where $T_{p}$ is the impeller torque, $\mathrm{N} \cdot \mathrm{m}$ and $\omega$ is the angular velocity of the impeller, $\mathrm{rad} / \mathrm{s}$.

According to Figure 8, the blades in Scheme I, Scheme II, and Scheme IV have no angle deviation. With an increase in blade angle, the head and efficiency curve shift to the large flow condition. Hence, the workability of each blade is enhanced, and the best efficiency of the pump device for each scheme basically remains unchanged.

Comparing Scheme II and Scheme III reveals that the performance curves of the pump device in Scheme II and Scheme III are basically identical. From the perspective of energy performance comparison, the workability of the blades in Scheme II (two blades with an angle of $0^{\circ}$ and two blades with an angle of $+4^{\circ}$ ) and that in Scheme III (four blades with an angle of $+2^{\circ}$ ) are basically consistent.

4.2. Blade Surface Pressure Analysis. The blade pressure surface of each scheme is shown in Figure 9, and the blade 


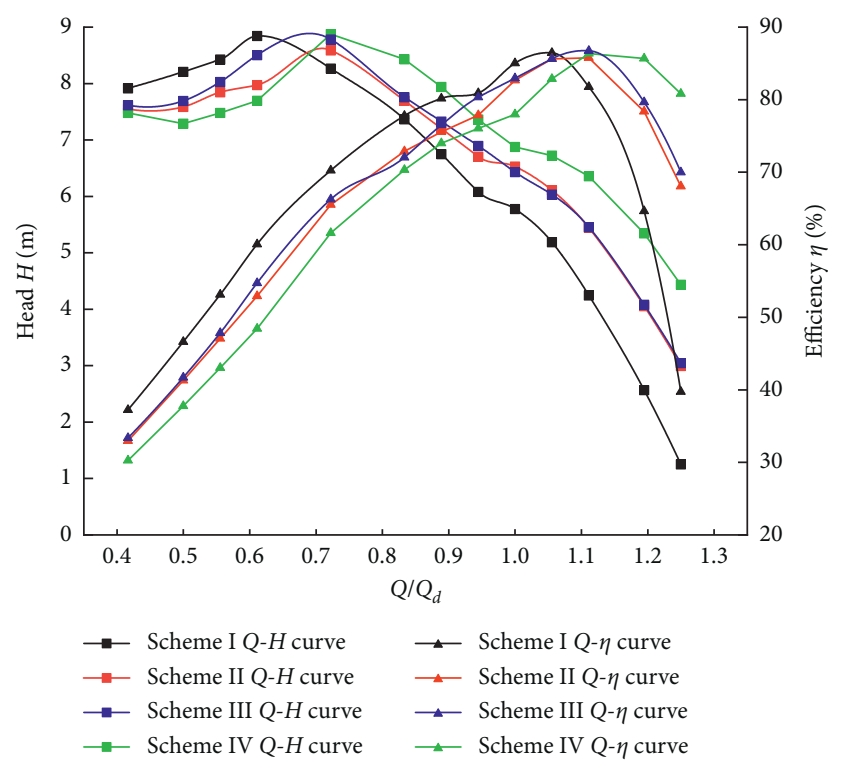

Figure 8: Performance curve of each scheme.

suction blade is shown in Figure 10. The pressure gradients of the four blade pressure surfaces in Scheme I, Scheme III, and Scheme IV are uniform. The pressure on the blade suction surface decreases first and then increases from the blade inlet to the blade outlet. When $Q=Q_{\text {bep }}$ and with the angle of the pump blades in Schemes I, III, and IV gradually increasing, the range of the high-pressure area at the shroud of the blade pressure surface broadens, along with the range of the low-pressure area on the suction surface of each blade. Hence, the workability of the blade is enhanced. However, under the same flow condition, with the increase in blade angle, the low-pressure area at the blade suction surface gradually increases. This result indicates that the cavitation performance of the pump is reduced and that cavitation is likely to occur when the blades operate at a large angle.

According to Figures 9(b) and 10(b), when the angles of the four blades are inconsistent, the pressure distribution trends of the symmetrical blades are consistent. Comparing the pressure distributions on the blade surfaces in Scheme II and Scheme III reveals that although the energy performances of the impeller in the two schemes are similar, the pressure distributions on the pressure and suction surface of each blade are inconsistent. In Scheme II, due to the deviation of each blade angle, the adjacent blades influence one another. Blade nos. 2 and 4 with large angles show a large pressure difference and strong workability. At the same time, blade nos. 1 and 3 with small angles show a small pressure difference and weak workability. In Scheme III, the pressure distributions on the blade surfaces are uniform, and the workability levels are equal.

4.3. Analysis of Flow Field in Impeller. To compare the flow characteristics in the impeller passage of the axial-flow pump and investigate the flow rule in the impeller, this study establishes a velocity streamline at the blade spans of $0.1,0.5$, and 0.9. The blade span distribution is shown in Figure 11.
Figures 12 and 13 show the velocity streamline for the blade spans in Scheme II and Scheme III under the optimal working condition. At the leading edge of the blade, the inflow is inclined to the blade suction surface because of the change of inlet attack angle, thus leading to a relatively higher velocity concentrating on the leading edge of the blade suction surface. In Scheme II, when the angles of two blades (nos. 2 and 4) with deviation change to $4^{\circ}$ symmetrically, the uniformity of the flow field between each blade passage worsens, and the distribution of velocity at the leading edge of the suction surface between the adjacent blades is inconsistent. At the same time, the velocity of a $4^{\circ}$ blade angle is higher than that of a $0^{\circ}$ blade angle; in the former case, cavitation is relatively easy to induce.

At the span $=0.1$, obvious leakage occurs at the outlet of the impeller between the two passages with angle deviation. It is due to the high-speed flow from the suction tailing of the blade with angle deviation to the front of the next adjacent blade (nos. 1 and 3), and it exerts an impact on the flow field in the passage. As the angles of blades no. 1 and no. 3 are small, the blade passage flow rate is relatively low, and the flow is a mix of low-speed flow and high-speed flow. This condition exacerbates the passage of turbulent disturbance, reduces the flow rate, and forms unstable flow states such as leakage. At the span $=0.5$ and 0.9, the low-speed zone develops into a $0^{\circ}$ blade pressure surface, which affects the workability of the blade shroud.

The flow fields at the blade span in Scheme III are compared, and the results reveal that the flow field in the pump is uniform and stable with good periodicity between each blade passage. Moreover, no unstable flow, such as leakage and vortex, is observed.

4.4. Analysis of Shaft Power Analysis. Figure 14 shows the power variation curves of the blade under the optimal working conditions within the two impeller rotation cycles. With the rotation of the impeller, the axial power curve in Scheme III shows periodic changes with small fluctuations and obvious regularity. The maximum amplitude of the variation is $0.036 \mathrm{~kW}$, and the average axial power is $24.339 \mathrm{~kW}$. The axial power in Scheme II is unstable and fluctuates greatly during the impeller's rotation period. The maximum amplitude of the variation is $0.056 \mathrm{~kW}$, which is 1.56 times that in Scheme III. The average axial power is $24.341 \mathrm{~kW}$. In Scheme II, the angle deviation of each blade leads to uneven pressure changes in each impeller passage and uneven forces on each blade surface. This condition consequently results in large shift power fluctuations. Such fluctuations affect the service safety of the impeller and cause fatigue damage and vibration in the blade.

\section{Analysis of Pressure Pulsation Characteristics}

A total of 12 monitoring points are uniformly arranged from the shroud to the hub in the impeller inlet, impeller outlet, and guide vane outlet to further analyze the influence of blade angle deviation on the pressure pulsation performance 

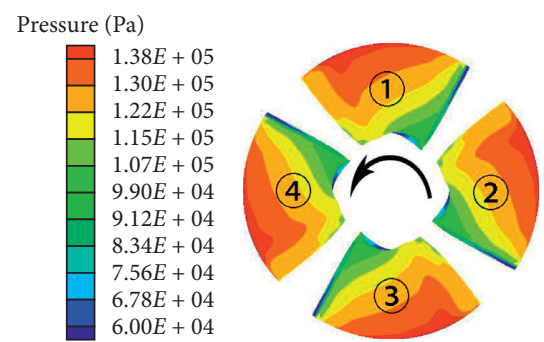

(a)

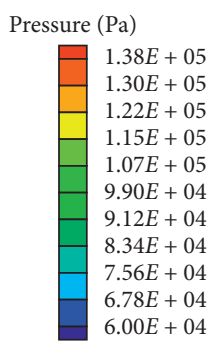

$1.38 E+05$
$1.30 E+05$
$1.22 E+05$
$1.15 E+05$
$1.07 E+05$
$9.90 E+04$
$9.12 E+04$
$8.34 E+04$
$7.56 E+04$
$6.78 E+04$
$6.00 E+04$

ressure $(\mathrm{Pa})$

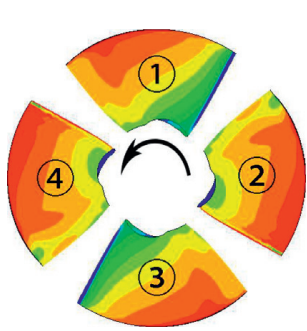

(b)

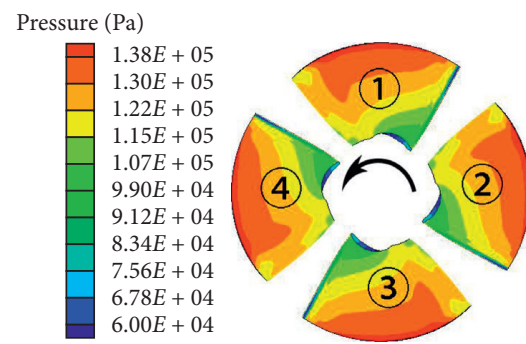

(c)

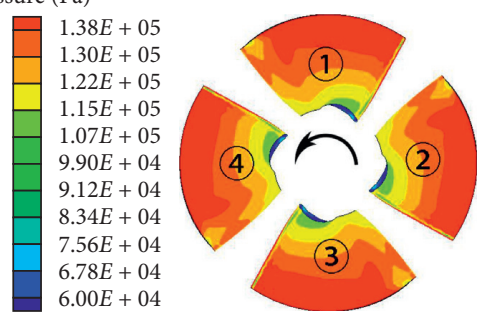

(d)

FiguRe 9: Pressure distribution of impeller pressure surface in different schemes $\left(Q=Q_{\text {bep }}\right)$. (a) Scheme I. (b) Scheme II. (c) Scheme III. (d) Scheme IV.

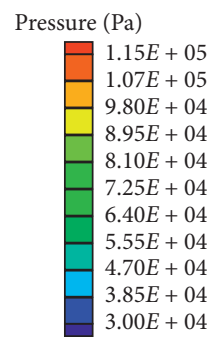

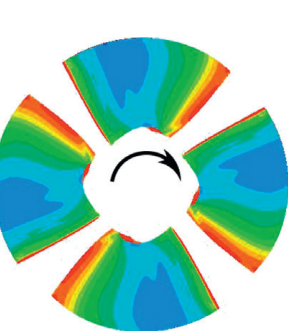

(a)
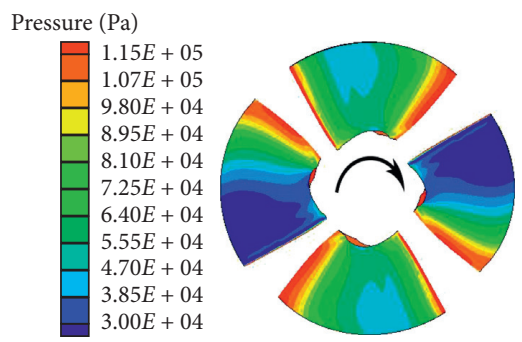

(b)

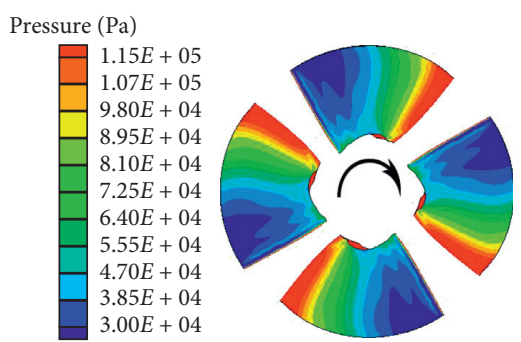

(d)

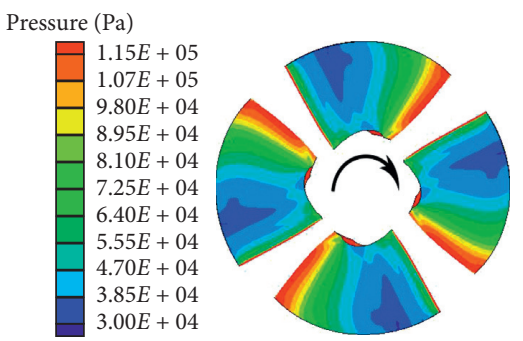

(c)

Scheme IV.

of the axial-flow pump device. The monitoring points $\mathrm{P} 1-\mathrm{P} 4$ are set at the impeller inlet, the monitoring points $\mathrm{P} 5-\mathrm{P} 8$ are set at the impeller outlet, and the monitoring points P9-P12 are set at the guide vane outlet (Figure 15). The rotating frequency $f_{n}$ and pressure pulsation coefficient $C_{p}$ are used to characterize the internal pressure pulsation characteristics of the pump device.

The rotating frequency equation is shown as follows:

$$
f_{n}=\frac{60 F}{n}
$$

where $F$ is the frequency obtained by fast Fourier transform, $\mathrm{Hz}$ and $n$ is rotation speed, $\mathrm{r} / \mathrm{min}$.

The pressure pulsation coefficient equation is shown as follows:

$$
C_{p}=\frac{P-P_{\text {ave }}}{0.5 \rho u^{2}},
$$

where $P$ is the instantaneous pressure, $\mathrm{Pa} ; P_{\text {ave }}$ is the average pressure, $\mathrm{Pa}$; and $u$ is the circumferential velocity of impeller, $\mathrm{m} / \mathrm{s}$. 


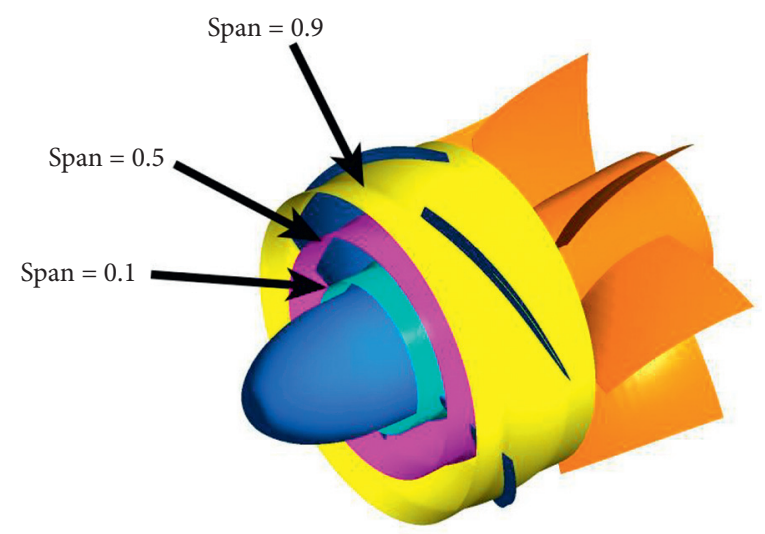

FIgURE 11: Span distribution.

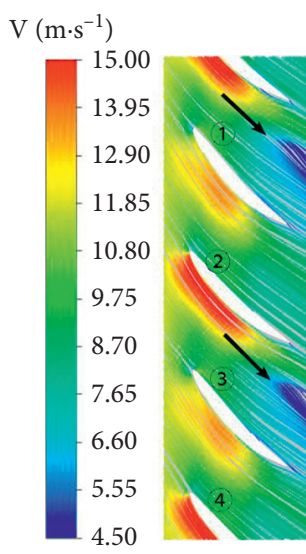

(a)

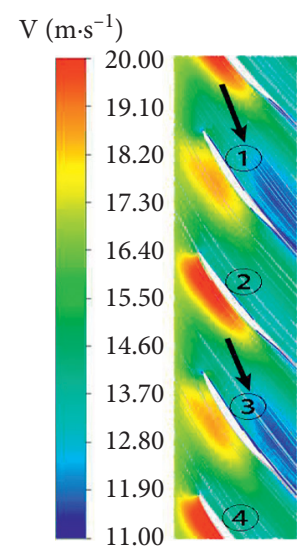

(b)

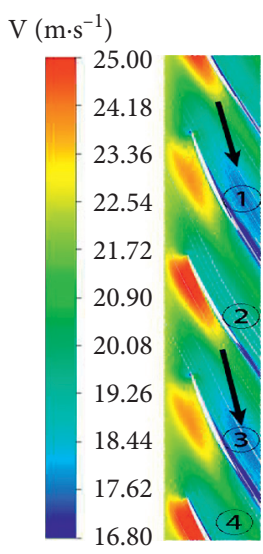

(c)

FIGURE 12: The velocity streamline of each span in Scheme II $\left(Q=Q_{\text {bep }}\right)$. (a) Span $=0.1$. (b) Span $=0.5$. (c) $\operatorname{Span}=0.9$.

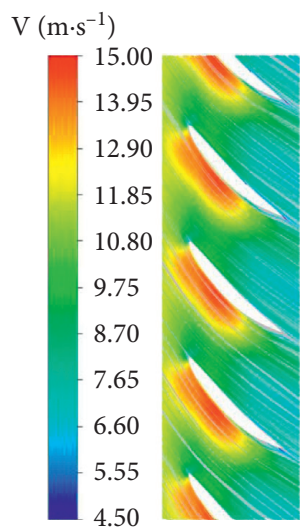

(a)

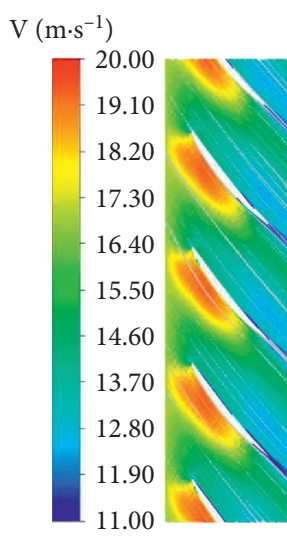

(b)

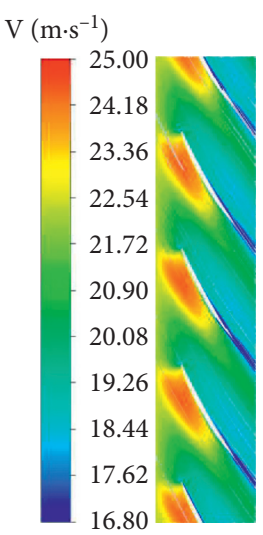

(c)

FIGURE 13: The velocity streamline of each span in Scheme III $\left(Q=Q_{\text {bep }}\right)$. (a) $\operatorname{Span}=0.1$. (b) $\operatorname{Span}=0.5$. (c) $\operatorname{Span}=0.9$.

5.1. Pressure Pulsation Characteristics of Impeller Inlet. According to the time domain characteristics of each monitoring point at the impeller inlet in Figure 16, the impeller inlet pressure pulsation in Scheme II and that in Scheme III show obvious periodicity. Moreover, the pressure pulsation decreases gradually from the shroud to the hub, but the periodicity of the two schemes is inconsistent. Scheme III shows the good periodicity of the pressure pulsation at each monitoring point and a uniform pressure fluctuation. The pressure pulsation in Scheme II is obviously higher than that in Scheme III. The peak-to-peak pulsation values of the monitoring points from the shroud to the hub 


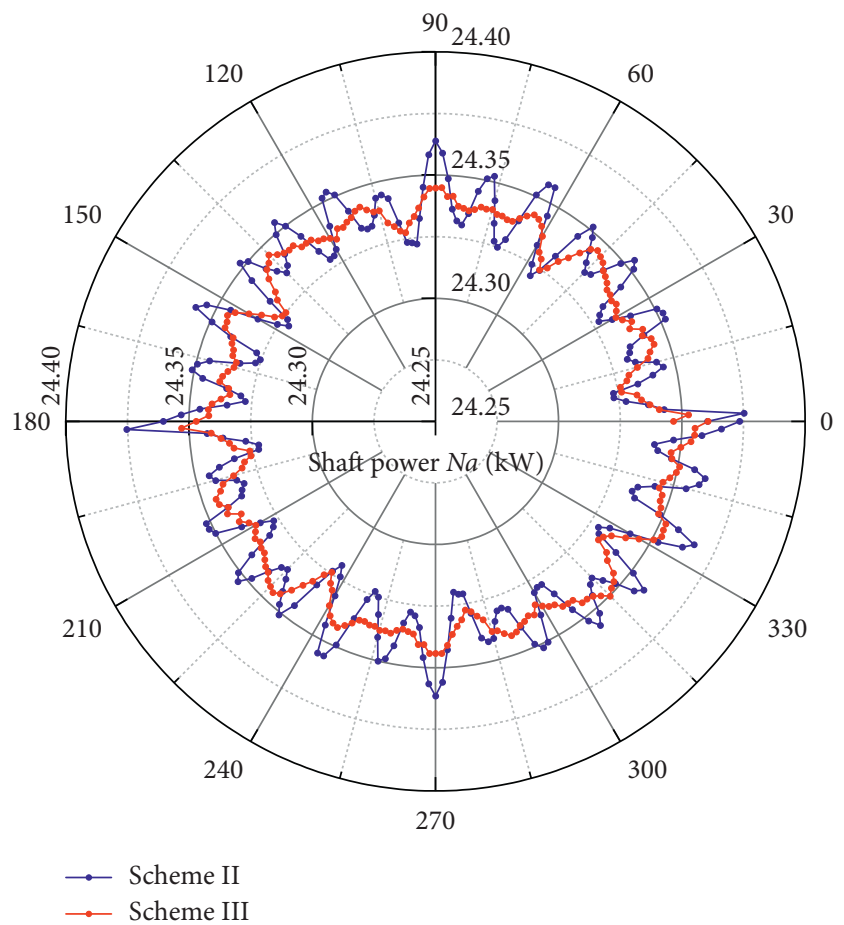

FIgURE 14: Power variation curve of blade shaft $\left(Q=Q_{\text {bep }}\right)$.

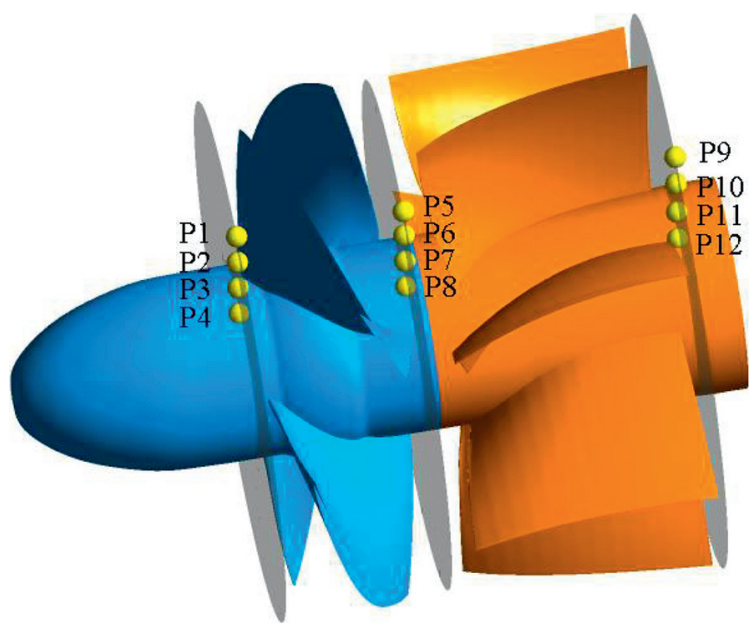

FIGURE 15: Distribution of monitoring points.

are about $1.53,1.54,1.56$, and 1.61 times those in Scheme III. These results indicate that the angle deviation in each blade increases the pressure pulsation of the impeller inlet.

According to Figure 17, the frequency domain characteristics of the pressure pulsation in Scheme II and Scheme III are 4 times the rotation frequency $\left(\mathrm{RF}=f_{n}=23.56 \mathrm{~Hz}\right)$, which is the blade passing frequency $\left(\mathrm{BPF}=4 f_{n}=94.22 \mathrm{~Hz}\right)$. The amplitude of pressure pulsation increases gradually from the hub to the shroud. The harmonic frequency in Scheme III is 8 times the RF. The harmonic frequency in Scheme II is 2 times the RF. In Scheme II, the pulsation amplitudes of the harmonic frequency from the shroud to the hub account for $70.13 \%, 71.92 \%, 79.35 \%$, and $90.81 \%$ of the main frequency amplitudes, respectively. This low-frequency pulsation greatly influences the stable operation of the pump and induces impeller vibration in severe cases.

5.2. Pressure Pulsation Characteristics of Impeller Outlet. The time domain characteristics of each monitoring point at the impeller outlet shown in Figure 18 indicate that the regularity of the impeller outlet's pressure pulsation in Scheme II and that in Scheme III are worse than those of the impeller inlet. Moreover, the peak pulsation value is obviously lower than that of the impeller inlet, and it accounts for less than $18 \%$ of the peak value at the impeller inlet. The 


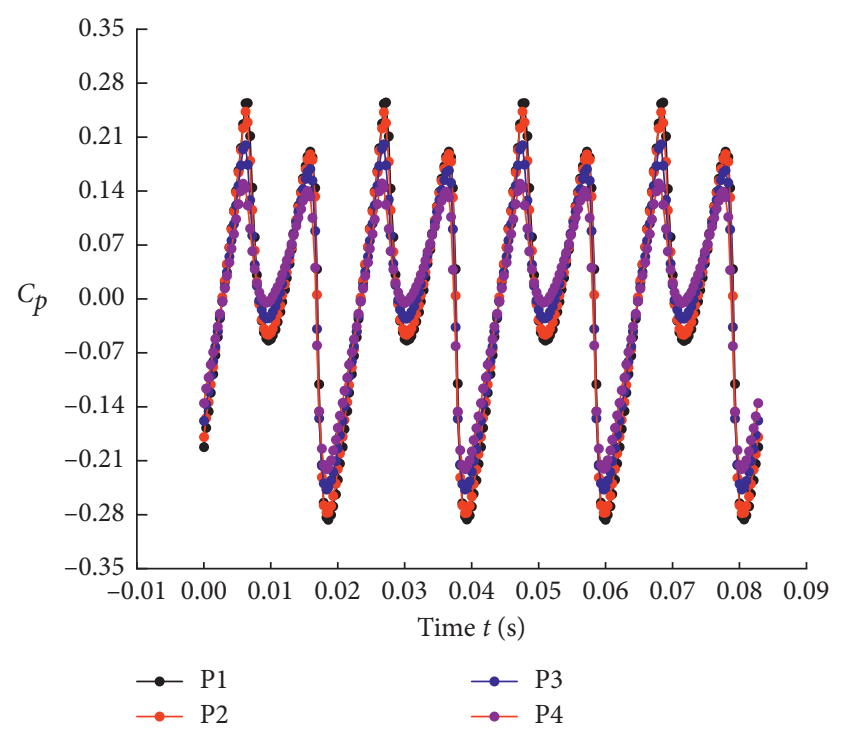

(a)

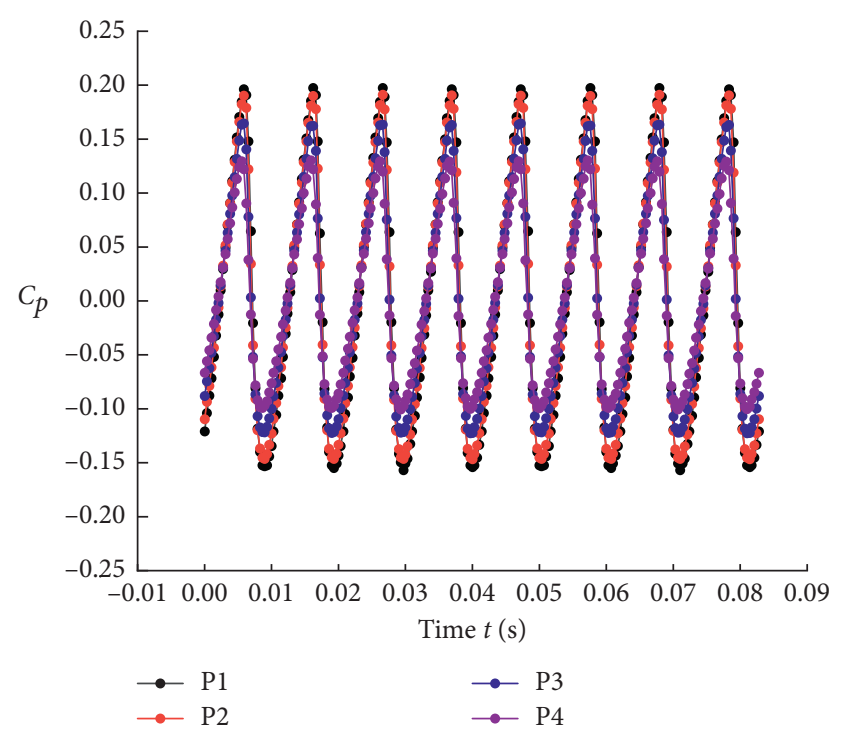

(b)

FIgURE 16: Time domain of each monitoring point at the impeller inlet $\left(Q=Q_{b e p}\right)$. (a) Scheme II. (b) Scheme III.

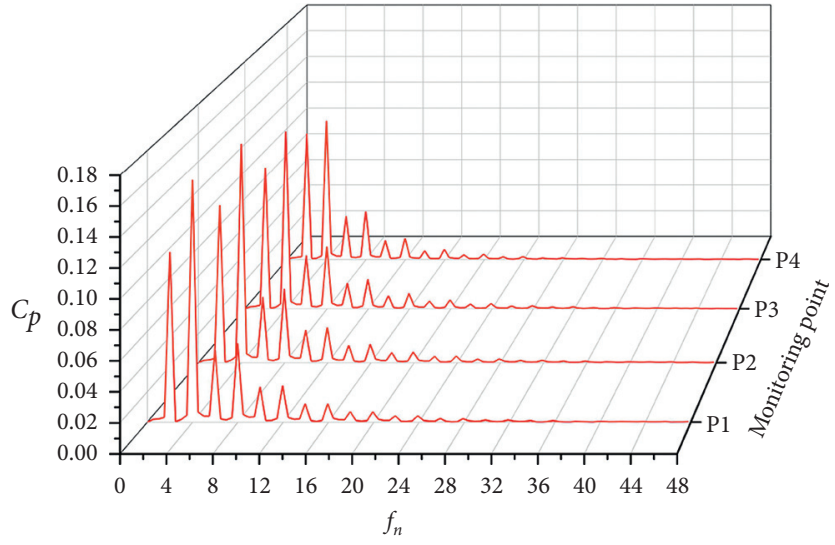

(a)

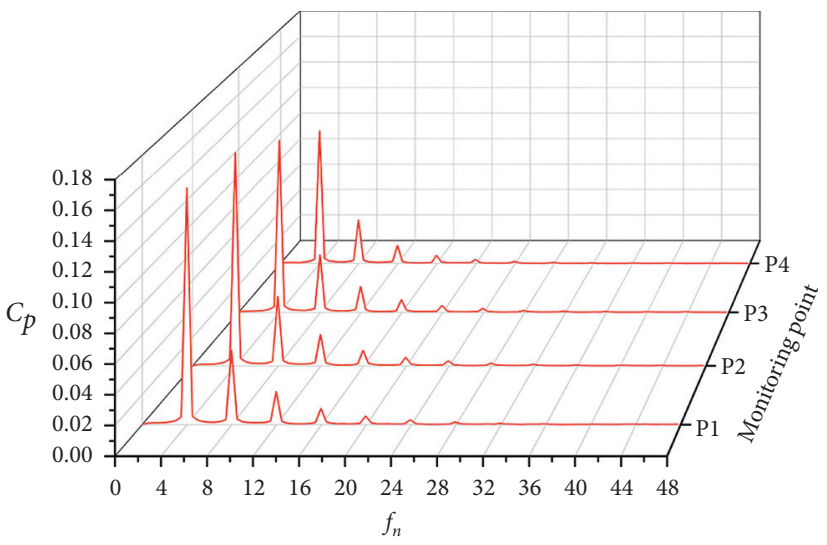

(b)

FIGURE 17: Frequency domain of each monitoring point at the impeller inlet $\left(Q=Q_{\text {bep }}\right)$. (a) Scheme II. (b) Scheme III.

pressure pulsation decreases gradually from the shroud to the hub. The peak-to-peak values of the pressure pulsation coefficients at each monitoring point in Scheme II are about $2.93,2.63,2.46$, and 5.48 times those in Scheme III, respectively. The fluctuation of the pressure pulsation amplitude at the hub is obvious and is mainly due to the unstable flow, such as leakage, which corresponds to the flow field mentioned previously.

According to Figure 19, different from the pressure pulsation at the inlet of the impeller, the main frequency at the outlet section of the impeller in the Scheme II is twice the $\mathrm{RF}$; the harmonic frequency is the BPF; and the main frequency amplitudes from the shroud to the hub are 0.048, $0.04,0.031$, and 0.016 , respectively. The low-frequency pressure pulsation is dominant, and it may be caused by the large low-frequency fluctuation induced by the unstable flow, such as leakage and vortex. The main frequency of Scheme III is the BPF and the harmonic frequency is 8 times the RF. Hence, the main frequency at the outlet of the impeller is still affected by the blade, and the maximum pressure pulsation amplitude of the frequency domain is 0.017 , which accounts for $35.42 \%$ of that in Scheme II. Compared with the frequency domain characteristics of the impeller inlet, the main frequency of the pressure pulsation induced by blade angle deviation at the impeller outlet is transformed into low frequency (twice the RF). Such low frequency easily causes the vibration of the impeller and endangers the safety and stability of the pump.

\subsection{Pressure Pulsation Characteristics of Guide Vane Outlet.}

The time domain of each monitoring point at the outlet of the guide vane in Figure 20 shows that the pressure pulsation at the outlet of the guide vane is obviously smaller than that in the impeller. The pressure fluctuations under the two schemes are periodic, but the peak pressure changes in the 


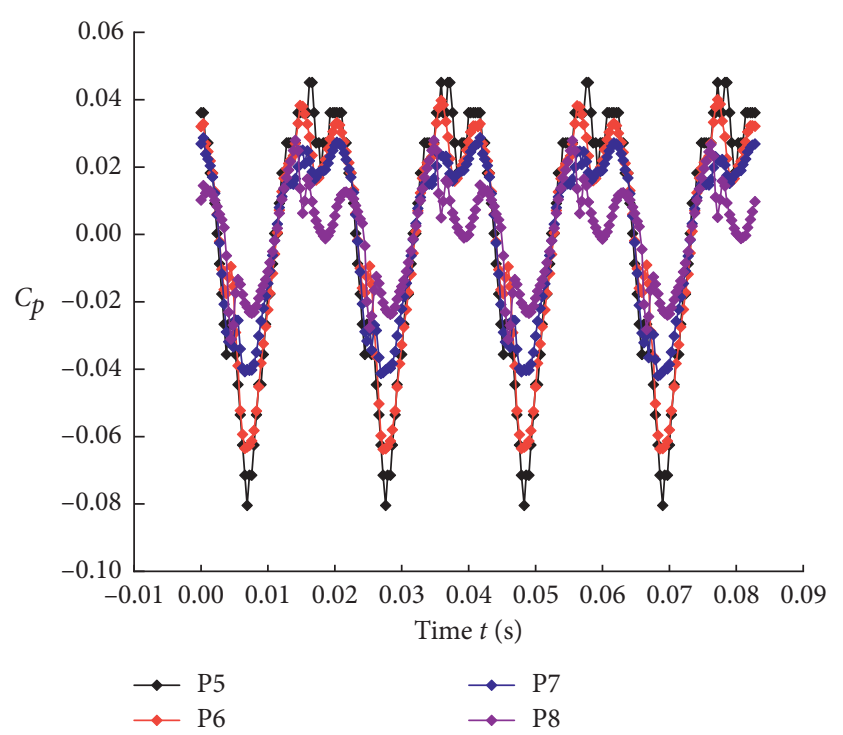

(a)

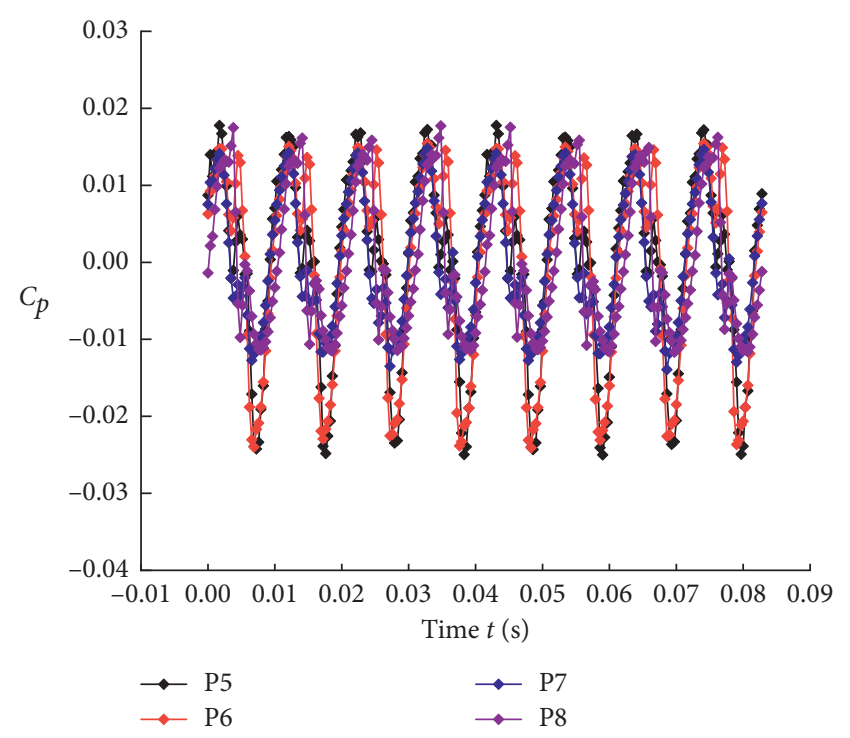

(b)

Figure 18: Time domain of each monitoring point at the impeller outlet $\left(Q=Q_{\text {bep }}\right)$. (a) Scheme II. (b) Scheme III.

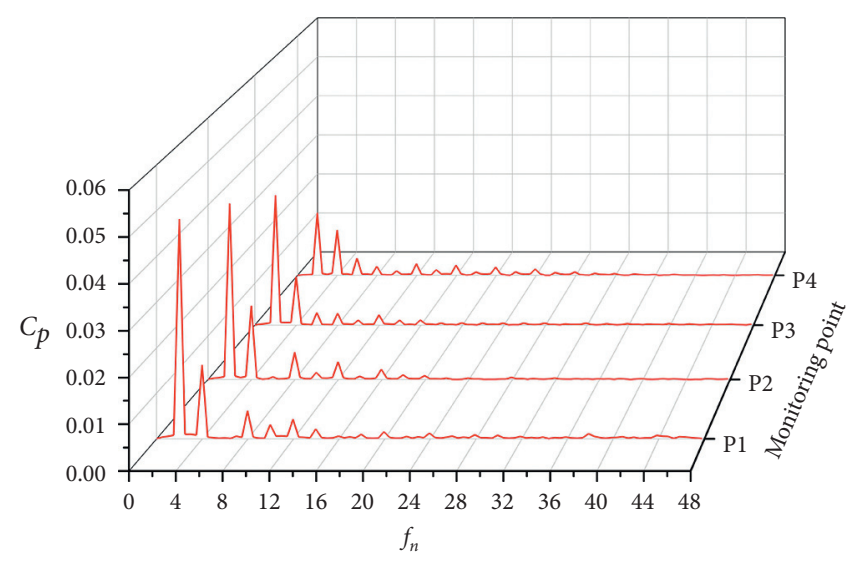

(a)

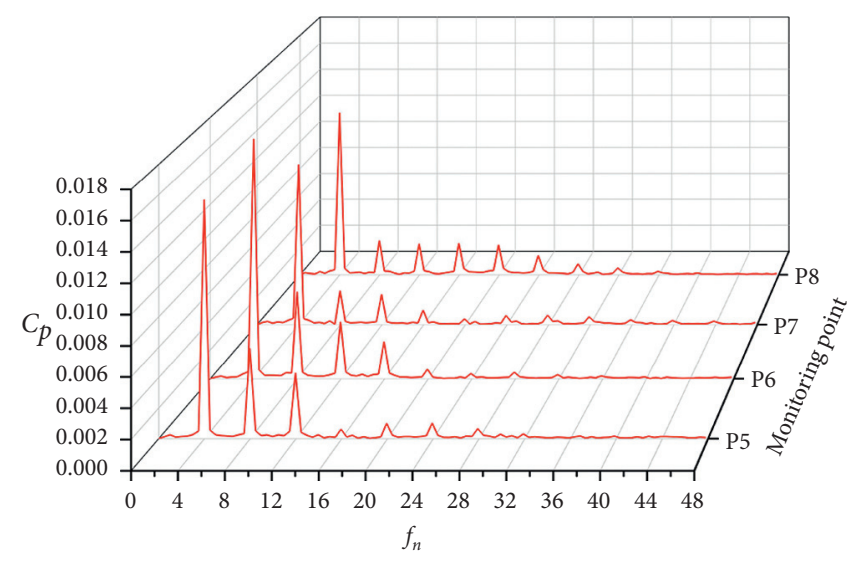

(b)

FIGURE 19: Frequency domain of each monitoring point at the impeller outlet $\left(Q=Q_{\text {bep }}\right)$. (a) Scheme II and (b) Scheme III.

two schemes are disordered and have poor regularity. Moreover, the second harmonic changes are obvious. Although the uneven pressure fluctuation caused by blade angle deviation decreases more obviously than that in the impeller inlet after the recovery effect of the guide vane, the pressure pulsation of Scheme II is still 2-3 times that of Scheme III.

According to Figure 21, the main frequency at the outlet of the guide vane in Scheme II is still twice the RF; the harmonic frequency is the BPF. The main frequency pulsation corresponding of guide vane outlet only accounts for $4.37 \%$ of that of the impeller inlet and for $14.55 \%$ of that of the impeller outlet. In Scheme III, except for the main frequencies of the monitoring point $\mathrm{P} 9$ of the shroud (i.e., $\mathrm{BPF}$ ), the main frequencies of the other monitoring points are 8 times the RF, and the harmonic frequencies are the
BPF. The pressure pulsation caused by blade angle deviation exerts a great influence on the pressure pulsation characteristics in the pump device. The main frequency of pressure pulsation at the outlet of the guide vane is still low frequency (twice the RF), which indicates that the whole operation condition of the pump is in a low-frequency pulsation. This poses great danger to pump safety.

By comparing the pressure pulsation at the sections in Scheme II and Scheme III, this study finds that the pressure pulsation amplitude at the inlet of the impeller is the largest. Blade angle deviation induces uneven pressure fluctuation. The amplitude of pressure pulsation is 1.5-3 times that of the blade without angle deviation at different positions in the pump device, and the regularity of pressure fluctuation in each impeller rotation period is poor. At the same time, the frequency of each section of Scheme II is mainly low 


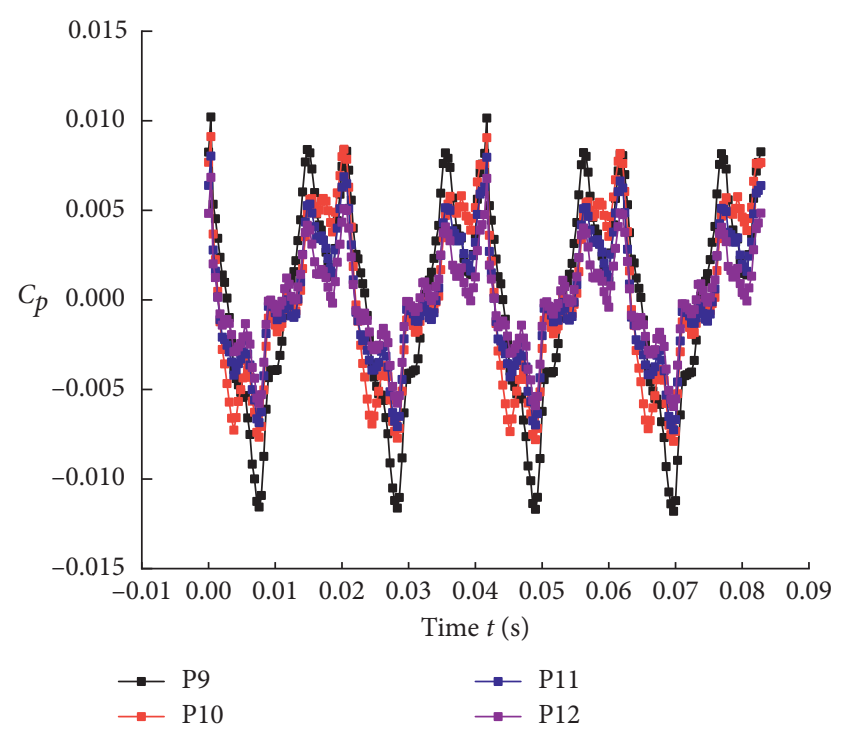

(a)

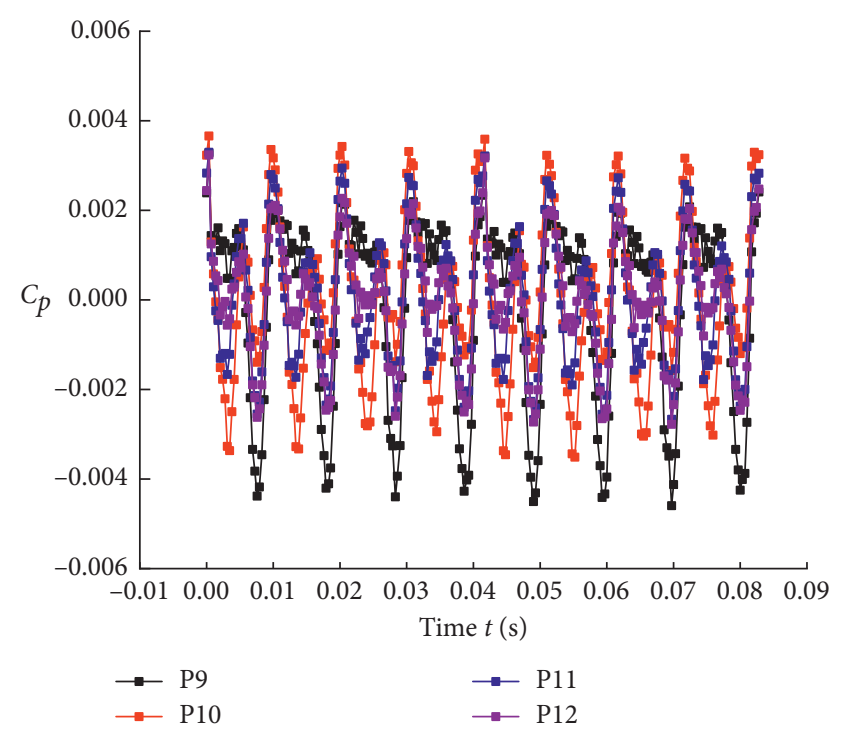

(b)

FIgURE 20: Time domain of each monitoring point at the guide vane outlet $\left(Q=Q_{b e p}\right)$. (a) Scheme II. (b) Scheme III.

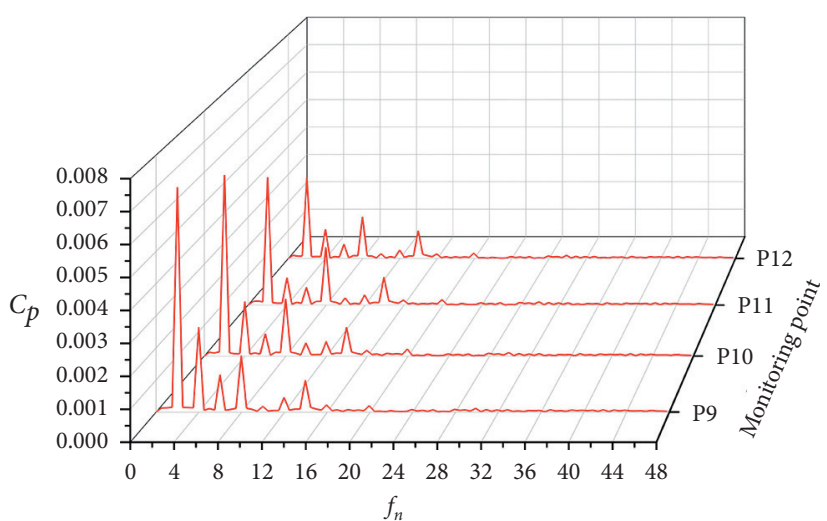

(a)

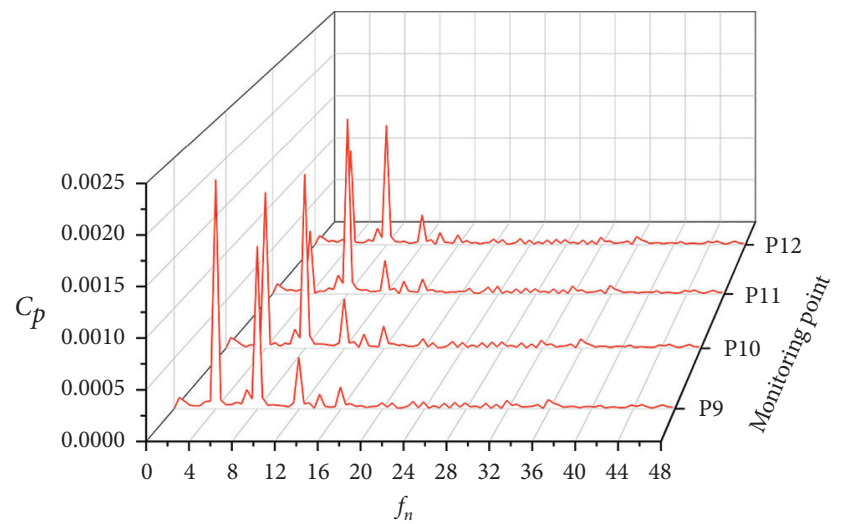

(b)

FIgURE 21: Frequency domain of each monitoring point at the guide vane outlet $\left(Q=Q_{\text {bep }}\right)$. (a) Scheme II. (b) Scheme III.

frequency. On the one hand, this low-frequency fluctuation causes an uneven force on the impeller surface and fatigue damage in the structure. On the other hand, it can easily cause pump resonance when the frequency of pressure pulsation is close to the natural frequency of the pump device.

\section{Conclusions}

In this study, the hydraulic characteristics and pressure pulsation of an axial-flow pump under the condition of blade angle deviation are analyzed by means of the numerical simulation and model test. The main conclusions are as follows:

(1) With no deviation of the blade angle, as the blade angle increases, the head of the pump increases.
Moreover, the efficiency and head curves shift to large flow conditions. When the blade angle is deviated, the adjacent blade exerts a mutual influence. As the blade angle is large, the pressure difference on the blade surface is large. As the blade angle is small, the pressure difference on the blade surface is small. The pressure distributions of the pressure and suction blade surfaces in each blade are inconsistent.

(2) When the blade angle is deviated, the flow field in the pump is not uniform. Because of the change of the inlet attack angle, the flow velocity on the leading edge of the blade suction with a large angle is high, and cavitation easily occurs. The uniformity of the flow field between each blade passage worsens, and the velocity distribution at the leading edge is inconsistent. 
(3) When the blade angle is deviated, the shaft power is unstable during the rotation period of the impeller. Moreover, the main frequency of the impeller inlet is the BPF, and that at the outlet of the impeller and the outlet of the guide vane is twice the RF, which is mainly low-frequency pulsation. Moreover, the pulsation amplitude at different positions in the pump device is obviously higher than that of the blade without angle deviation. Without any deviation in the blade angle, the shaft power changes periodically, showing obvious regularity and causing the small fluctuation. The main frequency of pressure pulsation at each section is the integer times of the BPF.

The blade angle deviation in the impeller exerts remarkable effects on the flow fields and vibration in the axialflow pump. Hence, it requires extensive research attention.

\section{Abbreviations}

\begin{tabular}{|c|c|}
\hline$D:$ & Impeller diameter, $\mathrm{mm}$ \\
\hline$Q_{d}:$ & Design flow, L/s \\
\hline Q: & Flow, L/s \\
\hline$d_{h}:$ & Hub ratio \\
\hline$\rho:$ & The density of flow, $\mathrm{kg} / \mathrm{m}^{3}$ \\
\hline$P_{\text {out }}:$ & $\begin{array}{l}\text { Total pressure at the outlet section of pump } \\
\text { device, } \mathrm{Pa}\end{array}$ \\
\hline$P_{\text {in }}:$ & $\begin{array}{l}\text { Total pressure at the inlet section of pump } \\
\text { device, } \mathrm{Pa}\end{array}$ \\
\hline$g:$ & Local acceleration of gravity, $\mathrm{m} / \mathrm{s}^{2}$ \\
\hline$H:$ & Head, $\mathrm{m}$ \\
\hline$\eta:$ & Efficiency, \% \\
\hline$T_{p}:$ & The impeller torque, $\mathrm{N} \cdot \mathrm{m}$ \\
\hline$\omega:$ & The angular velocity of the impeller, $\mathrm{rad} / \mathrm{s}$ \\
\hline$F:$ & $\begin{array}{l}\text { The frequency obtained by fast Fourier } \\
\text { transform, } \mathrm{Hz}\end{array}$ \\
\hline$n:$ & Rotation speed, r/min \\
\hline$P:$ & The instantaneous pressure, $\mathrm{Pa}$ \\
\hline Pave: & The average pressure, $\mathrm{Pa}$ \\
\hline$u:$ & The circumferential velocity of impeller, $\mathrm{m} / \mathrm{s}$ \\
\hline bep: & Best efficiency point \\
\hline$C_{p}:$ & Pressure pulsation coefficient \\
\hline$f_{n}:$ & $\mathrm{RF}$ rotating frequency, $\mathrm{Hz}$ \\
\hline BPF: & Blade passing frequency, $\mathrm{Hz}$ \\
\hline$G_{k}, G_{b}$ & $\begin{array}{l}\text { The generation term of turbulent kinetic energy } \\
k\end{array}$ \\
\hline$Y_{M}:$ & The contribution of pulsation expansion \\
\hline $\begin{array}{l}C_{1 \varepsilon}, C_{2 \varepsilon} \\
C_{3 \varepsilon}:\end{array}$ & Empirical constants \\
\hline$\sigma_{k}, \sigma_{\varepsilon}:$ & $\begin{array}{l}\text { The Prandtl numbers corresponding to } \\
\text { turbulent kinetic energy } k\end{array}$ \\
\hline$\sigma_{\varepsilon}:$ & $\begin{array}{l}\text { The Prandtl numbers corresponding to } \\
\text { dissipation rate } \varepsilon\end{array}$ \\
\hline$S_{k}, S_{\varepsilon}:$ & User-defined source terms \\
\hline$u_{i}:$ & $\begin{array}{l}\text { The fluid velocity component in the direction of } \\
\text { the coordinate } x_{i}, \mathrm{~m}^{2} / \mathrm{s}\end{array}$ \\
\hline$t:$ & The time, $s$ \\
\hline s. & A generalized source term. \\
\hline
\end{tabular}

\section{Data Availability}

All the data in this paper are obtained by physical experiment and numerical simulation, respectively, and the data used to support the findings of this study are available from the corresponding author upon request.

\section{Conflicts of Interest}

The authors declare that they have no conflicts of interest regarding the publication of this paper.

\section{Authors' Contribution}

Data curation was carried out by Lijian Shi, Penglan Huang, and Yao Yuan. Formal analysis was performed by Fangping Tang. Jun Zhu wrote the original draft. Wenpeng Zhang, Haiyu Liu, and Xiaowen Zhang reviewed and edited the article.

\section{Acknowledgments}

This work was supported by the National Natural Science Foundation of China (Grant no. 51376155); the Natural Science Foundation of Jiangsu Province of China (Grant no. BK20190914); China Postdoctoral Science Foundation Project (2019M661946); the Natural Science Foundation of Jiangsu Higher Education Institutions of China (Grant no. 19KJB570002); the National Science Foundation of Yangzhou of China (Grant no. YZ2018103); and the Priority Academic Program Development of Jiangsu Higher Education Institutions (Grant number PAPD).

\section{References}

[1] C. Liu, "Researches and developments of axial-flow pump system," Transactions of the Chinese Society for Agricultural Machinery, vol. 46, no. 6, pp. 49-59, 2015, in Chinese.

[2] H. L. Wang, B. Long, C. Wang et al., "Effects of the inpeller blade with a slot structure on the centrifugal pump performance," Energies, vol. 13, no. 7, pp. 1-17, 2020.

[3] Z. Qian, F. Wang, Z. Guo, and J. Lu, "Performance evaluation of an axial-flow pump with adjustable guide vanes in turbine mode," Renewable Energy, vol. 99, pp. 1146-1152, 2016.

[4] G. J. Peng, X. Huang, L. Zhou et al., "Soild-liquid two-phase flow and wear analysis in a large-scale centrifugal slurry pump," Engineering Failure Analysis, vol. 114, Article ID 104602, 2020.

[5] L. Shi, Y. Yuan, H. Jiao et al., "Numerical investigation and experiment on pressure pulsation characteristics in a full tubular pump," Renewable Energy, vol. 163, pp. 987-1000, 2021.

[6] S. Tang, S. Yuan, and Y. Zhu, "Deep learning-based intelligent fault diagnosis methods toward rotating machinery," IEEE Access, vol. 8, no. 1, pp. 9335-9346, 2020.

[7] L. Zhou, C. Han, L. Bai et al., "CFD-DEM bidirectional coupling simulation and experimental investigation of particle ejections and energy conversion in a spouted bed," Energy, vol. 211, Article ID 118672, 2020.

[8] X. K. He, Y. C. Zhang, C. Wang et al., "Influence of critical wall roughness on the performance of double-channel sewage pump," Energies, vol. 13, no. 2, 2020. 
[9] S. Tang, S. Yuan, and Y. Zhu, "Convolutional neural network in intelligent fault diagnosis toward rotatory machinery," IEEE Access, vol. 8, no. 1, pp. 86510-86519, 2020.

[10] H. L. Wang, Z. D. Qian, D. Zhang et al., "Numerical study of the normal impinging water jet at different impinging height, based on Wary-Agarwal turbulence model," Energies, vol. 13, no. 7, 2020.

[11] X. F. Wu, Y. D. Lu, M. G. Tan et al., "Effect of vane angle on axial flow pump running characteristics in saddle zone," Transactions of the Chinese Society of Agricultural Engineering (Transactions of the CSAE), vol. 34, no. 17, pp. 46-53, 2018, in Chinese.

[12] L. Sun, P. Guo, and X. Luo, "Numerical investigation on interblade cavitation vortex in a franics turbine," Renewable Energy, vol. 158, pp. 64-74, 2020.

[13] H. S. Shim and K. Y. Kim, "Design optimization of the impeller and volute of a centrifugal pump to improve the hydraulic performance and flow stability," Journal of Fluids Engineering, vol. 142, no. 10, 2020.

[14] H. L. Wang, B. Long, Y. Yang, Y. Xiao, and C. Wang, "Modelling the influence of inlet angle change on the performance of submersible well pumps," International Journal of Simulation Modelling, vol. 19, no. 1, pp. 100-111, 2020.

[15] Z. J. Wu, C. Hou, W. K. Liang et al., "Effect of blade installation angle on cavitation performance of axial flow pump," Chinese Journal of Hydrodynamics, vol. 35, no. 3, pp. 227-284, 2020, in Chinese.

[16] H. Yan, X. Z. Su, H. Z. Zhang et al., "Design approach and hydrodynamic characteristics of a novel bionic airfoil," Ocean Engineering, vol. 216, Article ID 108076, 2020.

[17] H. Ding, Z. Li, X. Gong, and M. Li, "The influence of blade outlet angle on the performance of centrifugal pump with high specific speed," Vacuum, vol. 159, pp. 239-246, 2019.

[18] C. Guo, M. Gao, J. Wang, Y. Shi, and S. He, "The effect of blade outlet angle on the acoustic field distribution characteristics of a centrifugal pump based on powell vortex sound theory," Applied Acoustics, vol. 155, pp. 297-308, 2019.

[19] B. Cui, C. Wang, Z. Zhu, and Y. Jin, "Influence of blade outlet angle on performance of low-specific-speed centrifugal pump," Journal of Thermal Science, vol. 22, no. 2, pp. 117-122, 2013.

[20] T. Wang, F. Y. Kong, S. S. Yang et al., "Effects of blade angle distributions on performance of pump as turbine," Transactions of the Chinese Society for Agricultural Machinery, vol. 46, no. 10, pp. 75-80, 2015, in Chinese.

[21] B. L. Cui, W. Q. Li, and C. L. Zhang, "Effect of blade trailing edge cutting angle on unstable flow and vibration in a centrifugal pump," Journal of Fluids Engineering, vol. 142, no. 10, 2020.

[22] Y. B. Liu, Y. D. Han, L. Tan et al., "Blade rotation angle on energy performance and tip leakage vortex in a mixed flow pump as turbine at pump mode," Energy, vol. 206, Article ID 118084, 2020.

[23] G. J. Peng, Q. Chen, L. Zhou et al., "Effect of blade outlet angle on the flow field and preventing overload in a Centrifugal Pump," Micromachines, vol. 11, no. 9, 2020.

[24] X. L. Feng and B. Y. Qiu, "Adjusting frequency of pump blade angles and optimal operation for large pumping station system," Advances in Mechanical Engineering, vol. 5, pp. 1-8, 2013.

[25] Q. S. Wei, X. H. Sun, A. Y. Shamseldin et al., "Impacts of blade inlet angle of diffuser on the performance of a submersible pump," Proceedings of the Institution of Mechanical Engineers
Part E Journal of Process Mechanical Engineering, vol. 234, no. 6, pp. 613-623, 2020.

[26] Y. S. Kim, M. W. Heo, H. S. Shim et al., "Hydrodynamic optimization for design of a submersible axial-flow pump with a swept impeller," Energies, vol. 13, no. 12, 2020.

[27] H. Bing and S. Cao, "Effects of blade rotation angle deviations on mixed-flow pump hydraulic performance," Science China Technological Sciences, vol. 57, no. 7, pp. 1372-1382, 2014.

[28] H. Bing, S. Cao, C. He, and L. Lu, "Experimental study of the effect of blade tip clearance and blade angle error on the performance of mixed-flow pump," Science China Technological Sciences, vol. 56, no. 2, pp. 293-298, 2013.

[29] L. Shi, W. Zhang, H. Jiao et al., "Numerical simulation and experimental study on the comparison of the hydraulic characteristics of an axial-flow pump and a full tubular pump," Renewable Energy, vol. 153, pp. 1455-1464, 2020.

[30] K. Kan, Y. Zheng, H. Chen et al., "Numerical simulation of transient flow in a shaft extension tubular pump unit during runaway process caused by power failure," Renewable Energy, vol. 154, pp. 1153-1164, 2020.

[31] Y. Yang, L. Zhou, W. D. Shi et al., "Interstage difference of pressure pulsation in a three-stageelectrical difference submersible pump," Journal of Petroleum Science and Engineering, vol. 196, Article ID 107653, 2020. 